\title{
CONTROLE QUÍMICO À BASE DE AZADIRACHTA INDICA EM BEMISIA SPP. NA CULTURA DA ABOBRINHA (CUCURBITA PEPO)
}

\author{
FARAH, Paulo José Kastein ${ }^{1}$ \\ ROSSI, Marta Maria ${ }^{2}$ \\ PINTO, Alexandre de Sene ${ }^{3}$
}

Recebido em: 2009-04-12

Aprovado em: 2009-09-25

ISSUE DOI: $10.3738 / 1982.2278 .209$

\begin{abstract}
RESUMO: A mosca-branca Bemisia spp. é um inseto sugador, considerado praga polífaga. Além dos danos provocados diretamente pela sucção de seiva e injeção de toxinas, é responsável pela transmissão de viroses e por alterações fisiológicas na planta, como o prateamento da abobrinha. O objetivo do trabalho foi avaliar diferentes doses de nim para o controle de Bemisia em abobrinha italiana, na época da seca. Foram testados seis tratamentos repetidos cinco vezes cada: (1) testemunha, sem tratamento; (2) aplicação de extrato de nim, na dosagem de $50 \mathrm{~mL}$ por $10 \mathrm{~L}$ de água; (3) aplicação de extrato de nim, na dosagem de $70 \mathrm{~mL}$ por $10 \mathrm{~L}$ de água; (4) aplicação de extrato de nim, na dosagem de $80 \mathrm{~mL}$ por $10 \mathrm{~L} \mathrm{de}$ água; (5) aplicação de extrato de nim, na dosagem de $90 \mathrm{~mL}$ por $10 \mathrm{~L}$ de água; (6) aplicação de thiametoxan (Actara - controle padrão), na dosagem de 1,5 g por $10 \mathrm{~L}$ de água. As avaliações foram realizadas em 12 plantas por parcela, aos 1, 3, 5 e 7 dias após as aplicações dos tratamentos. Um dia após a aplicação do extrato de nim, a testemunha diferiu estatisticamente de todos os tratamentos. Porém, o baixo poder residual do extrato e as concentrações usadas permitiram um aumento significativo do número de moscas brancas nas parcelas, no decorrer dos dias. A melhor dosagem do extrato de nim para o controle de mosca-branca na cultura da abobrinha italiana é de $900 \mathrm{~mL} / 100 \mathrm{~L}$ de água.
\end{abstract}

Palavras-chave: Mosca-branca. Hemiptera: Aleyrodidae, nim. Meliaceae. Abobrinha italiana cv Caserta

\section{Chemical control based on Azadirachta indica in Bemisia spp. in the cultivation of zucchini (Cucurbita pepo)}

SUMMARY: The whitefly Bemisia spp. is a sucking insect, considered a polyphagous pest. In addition to the damage caused directly by sucking sap and the injection of toxins, this insect is responsible for the transmission of viruses and physiological changes in the plant, as silver the zucchini. The objective was to evaluate different doses of Neem, for the control of Bemisia in italian zucchini, in the dry season. We tested six treatments repplicated five times: (1) control, without treatment, (2) application of the Neem extracts in dosage of $50 \mathrm{~mL}$ per 10 liters of water, (3) application of the Neem extracts in dosage of $70 \mathrm{~mL}$ per 10 liters of water; (4) application of the Neem extracts in dosage of 80 $\mathrm{mL}$ per 10 liters of water; (5) application of the Neem extracts in dosage of $90 \mathrm{~mL}$ per 10 liters of water; (6) implementation of Thiametoxan (Actara - standard control), the dosage of $1.5 \mathrm{~mL}$ per 10 liters of water. The evaluations were conducted in 12 plants per plot after 1, 3,5 and 7 days after the applications of treatments. One day after the application of the Neem extract, the control statistically differed of the all the treatments. However, the low residual power of the extract and the concentrations used allowed a significant increase in the number of whitefly in the plots, over the course of the day. The best dosage of the Neem extracts to control the whitefly in the culture of the Italian zucchini is $900 \mathrm{~mL} / 100 \mathrm{~L}$ water

\footnotetext{
${ }^{1}$ Engenheiro Agrônomo pelo Centro Universitário Moura Lacerda - CUML - em Ribeirão Preto - SP. paulofarah@gawab.com

${ }^{2}$ Professora Doutora, Faculdade "Dr. Francisco Maeda" - FAFRAM - Ituverava - SP e Centro Universitário Moura Lacerda - CUML - Ribeirão Preto - SP

${ }^{3}$ Professor Doutor, Centro Universitário Moura Lacerda - CUML - Ribeirão Preto - SP.
} 
Keywords: Whitefly. Hemyptera: Aleyrodidae, Neem, Meliaceae, Italian zucchini, Caserta Variety

\section{INTRODUÇÃO}

A abobrinha italiana, Cucúrbita pepo var. melopepo, também conhecida como "abóbora de árvore" ou "de moita", é um fruto pertencente à família Cucurbitaceae, originária do continente americano, do Peru até sul dos Estados Unidos (LANA et al, 2008), tendo usos bastante diversos na culinária. Em condições de campo, a cultura está sujeita ao ataque de diversas pragas, como brocas da ordem Lepidoptera, e também insetos sugadores, da ordem Hemiptera, como a Bemisia spp. Este inseto é considerado praga polífaga, por atacar plantas de diversas famílias, dentre elas Asteraceae, Solanaceae, Cucurbitaceae, Malvaceae, Euphorbiaceae e Fabaceae. Além dos danos provocados diretamente pela sucção da seiva e injeção de toxinas (GALLO et al, 2002), a mosca-branca também é responsável pela transmissão de viroses e alterações fisiológicas, como o prateamento da abobrinha.

As moscas-brancas são insetos erroneamente assim chamados, pois pertencem à ordem Hemiptera, subordem Sternorrhyncha, família Aleyrodidae (GALLO et al, 2002), e encontram-se entre os mais nocivos insetos infestando culturas no campo e em cultivo protegido em todo o mundo. Segundo Zucchi et al (1993), a taxonomia é baseada no pupário (morfologia do último ínstar de ninfa). Os pupários devem ser coletados juntos com a parte vegetal onde estão fixados, conservados em álcool $90 \%$ ou envelope entomológico, para serem montados e identificados. São insetos pequenos, de $1 \mathrm{~mm}$ de comprimento, com quatro asas membranosas recobertas por uma pulverulência branca. Os ovos são colocados na face inferior das folhas, ficando presos por um pedúnculo curto; após a eclosão, as ninfas passam a sugar a folha, geralmente na face inferior. Esta fase só se locomove inicialmente, fixando-se a seguir de maneira semelhante às cochonilhas. $\mathrm{O}$ ciclo completo é de cerca de 15 dias e as fêmeas possuem longevidade de 18 dias. A quantidade de ovos colocados por fêmea varia de acordo com o biotipo do inseto, entre 110 e 300 ovos (GALLO et al, 2002). Tanto ninfas como adultos alimentam-se da seiva do floema das plantas de centenas de espécies vegetais, causando danos diretos como manchamento, amarelecimento e queda das folhas, reduzindo o vigor das plantas, e também danos indiretos, devido à excreção de "honeydew" que favorece o desenvolvimento de fungos, e à transmissão de vírus (LENTEREN; NOLDUS, 1990; BYRNE; BELLOWS, 1991). 
A existência de biotipos de B. tabaci foi proposta a partir da década de 1950, após a descoberta de populações morfologicamente semelhantes, mas que exibiam aspectos biológicos diferentes em relação à planta hospedeira, à transmissão de vírus, à resistência a inseticidas e ao comportamento dos insetos (COSTA; BROWN 1991, BEDFORD et al 1994, BROWN et al 1995). Assim, o biotipo A não se reproduz bem em poinsétia ou bico-de-papagaio (Euphorbia pulcherrima), não induz o prateamento da folha em aboboreira e apresenta o padrão isoenzimático A, enquanto o biotipo B se reproduz intensamente em poinsétia e brócolis e induz o prateamento da folha da aboboreira, apresentando padrão isoenzimático B (LOURENÇÃO; NAGAI, 1994).

Possivelmente, pela importação de material vegetal, no início dos anos 90, o biotipo B foi introduzido no Brasil. Altas populações foram verificadas no Estado de São Paulo, na região de Campinas, em 1991 e 1992. Infestações severas foram observadas em plantas ornamentais e em cultivos de brócolis, berinjela, tomate e abóbora, ocasionando nessas duas últimas o amadurecimento irregular dos frutos e o prateamento das folhas, respectivamente (LOURENÇÃO; NAGAI, 1994). Logo a seguir, altas infestações foram também observadas em cultivos de tomate no Distrito Federal (FRANÇA et al, 1996).

Em presença de baixas populações, os prejuízos provocados pela mosca-branca são relacionados à transmissão de vírus, mas quando a densidade populacional é maior, além de vetor, Bemisia atua como praga (COSTA, 1976). Os prejuízos ocasionados por B. tabaci podem variar entre 20 e $100 \%$, dependendo da cultura, época e nível de infestação, entre outros fatores (BROWN; BIRD, 1992).

Para se fazer um controle correto da mosca-branca, deve-se fazer um levantamento populacional, que indicará o momento certo para a entrada de um método para controlá-la.

No tomateiro, no sistema de plantio por semeadura direta no campo, a amostragem de adultos pode ser feita após dez dias e a amostragem de ninfas, aos 24 dias. Nos outros sistemas de plantio a amostragem de adultos deve ser iniciada aos sete dias após o transplantio e a de ninfas, aproximadamente, aos 21 dias, com uma freqüência de quatro a sete dias (HAJI et al, 2005). Mattos (2001) recomenda que a amostragem de adultos da mosca-branca em tomateiro deve ser realizada na face inferior do folíolo apical da terceira folha do terço superior das plantas e a de ninfas, no folíolo apical de uma folha do terço mediano das plantas. Considera-se a amostra 
infestada quando forem encontrados um ou mais adultos e uma ou mais ninfas, na área delimitada do campo de visão da lupa (HAJI et al, 2005).

A mosca-branca vem sendo controlada por diversas práticas, entre elas as culturais (OLIVEIRA, 2001) e há alguns anos por meio do controle biológico com extratos de plantas, predadores naturais, fungos, etc. $\mathrm{O}$ controle cultural da moscabranca consiste em práticas como barreiras vivas, armadilhas, eliminação das plantas daninhas hospedeiras, eliminação de restos culturais, entre outros. Essas práticas são importantes para o controle, principalmente, em pequenas áreas de tomate e feijão, em virtude da baixa população deste inseto que também é um vetor de viroses (HILJE, 1995).

Usado indiscriminadamente por produtores, em todas as regiões do país, o controle químico deve ser feito respeitando o meio ambiente, ou seja, de forma racional e sempre usando produtos registrados para a cultura. Para o controle da mosca-branca, os produtos registrados estão discriminados a seguir (Tabela 1), sendo que somente dois produtos (*) são registrados especificamente para a cultura da abobrinha.

TABELA 1- Inseticidas registrados no Ministério da Agricultura, Pecuária e Abastecimento, para o controle da mosca-branca em diversas culturas, e na cultura da abobrinha $(*)$

\begin{tabular}{|c|c|c|c|c|}
\hline \multicolumn{5}{|c|}{ AGROTÓXICOS REGISTRADOS PARA Bemisia tabaci } \\
\hline Marca Comercial & Ingrediente Ativo & Class. Tox & Class. Amb & Registrante \\
\hline * Actara $250 \mathrm{WG}$ & tiametoxam & III & III & Syngenta Proteção de Cultivos Ltda. \\
\hline Applaud 250 & buprofezina & IV & III & Hokko do Brasil Ind. Quím. e Agrop. Ltda. \\
\hline Calypso & tiacloprido & III & III & Bayer CropScience Ltda. \\
\hline Confidor $200 \mathrm{SC}$ & imidacloprido & III & III & Bayer CropScience Ltda. \\
\hline * Confidor $700 \mathrm{GrDa}$ & imidacloprido & IV & III & Bayer CropScience Ltda. \\
\hline Counter $150 \mathrm{G}$ & terbufós & $\mathrm{I}$ & II & Basf S.A. \\
\hline Cruiser $700 \mathrm{WS}$ & tiametoxam & III & III & Syngenta Proteção de Cultivos Ltda. \\
\hline Deltaphos & deltametrina + triazofós & I & $\mathrm{I}$ & Bayer CropScience Ltda. \\
\hline Gaucho FS & imidacloprido & IV & III & Bayer CropScience Ltda. \\
\hline Hostathion $400 \mathrm{BR}$ & triazofós & $\mathrm{I}$ & II & Bayer CropScience Ltda. \\
\hline Karate Zeon $50 \mathrm{CS}$ & lambda-cialotrina & III & II & Syngenta Proteção de Cultivos Ltda. \\
\hline Keshet 25 CE & deltametrina & $\mathrm{I}$ & II & Agricur Defensivos Agrícolas Ltda. \\
\hline Mospilan & acetamiprido & III & II & Iharabras S.A. Indústrias Químicas \\
\hline Nomolt 150 & teflubenzurom & IV & II & Basf S.A. \\
\hline Ofunack $400 \mathrm{CE}$ & piridafentiona & III & II & Sipcam Agro S.A. \\
\hline Pirate & clorfenapir & III & II & Basf S.A. \\
\hline Pólo 500 PM & diafentiurom & $\mathrm{I}$ & II & Syngenta Proteção de Cultivos Ltda. \\
\hline Polytrin 400/40 CE & cipermetrina + profenofós & III & II & Syngenta Proteção de Cultivos Ltda. \\
\hline Provado & imidacloprido & IV & III & Bayer CropScience Ltda. \\
\hline Provado $200 \mathrm{SC}$ & imidacloprido & III & III & Bayer CropScience Ltda. \\
\hline Saurus & acetamiprido & III & II & Bayer CropScience Ltda. \\
\hline Talstar $100 \mathrm{CE}$ & bifentrina & III & III & FMC Química do Brasil Ltda. \\
\hline
\end{tabular}

FONTE: SIA - Sistema de informações sobre agrotóxicos (2008); ANVISA

(http://www4.anvisa.gov.br/AGROSIA/asp/frm_pesquisa_agrotoxico.asp).

${ }^{1} \mathrm{CT}=$ Classe Toxicológica. I - Extremamente tóxico (faixa vermelha); II - Altamente tóxico (faixa amarela); III - Moderadamente tóxico (faixa azul); IV - Pouco tóxico (faixa verde).

* Produtos registrados para o controle da mosca-branca na cultura da abobrinha. 
O nim, Azadirachta indica, é uma planta oriunda do sudeste asiático e do subcontinente indiano. Pertence à família Meliaceae, é uma planta de clima tropical, resistente à seca, de crescimento rápido, copa densa, chegando a alcançar $15 \mathrm{~m}$ de altura, podendo ser cultivada em regiões de clima quente e solos bem drenados (SOGLIA et al., 2007). A floração e a frutificação do nim ocorrem a partir do terceiro ano, dependendo das condições climáticas e do estágio de desenvolvimento da planta. A frutificação ocorre uma vez por ano, em condições ideais, e podem ser feitas até duas colheitas por ano (NEVES et al; 2003). Os frutos possuem forma oval medindo de 1,4 a 2,4 cm de comprimento, polpa doce amarelada e tegumento branco, contendo um óleo marrom no interior de uma semente (SCHUMUTTERER, 1990). As sementes de melhor qualidade são obtidas quando os frutos atingem a fase de maturação. De acordo com Ciociola; Martinez (2002) uma forma de coleta pode ser por meio de lonas que são colocadas nos pés das árvores onde as sementes caem naturalmente e são coletadas sem que os frutos entrem em contato com o solo, reduzindo assim a possibilidade do aparecimento de doenças.

Por ser de fácil obtenção e utilização, ter baixo custo e minimizar os problemas apresentados pelos produtos químicos sintéticos como surgimento de pragas secundárias e toxicidade, os inseticidas naturais de origem vegetal podem vir a se constituir em importantes agentes de controle. Extratos biologicamente ativos obtidos de folhas, frutos, sementes e tronco de A. indica são reconhecidos por suas propriedades inseticidas, sendo a azadiractina considerada o composto ativo mais potente da árvore de nim, isolada por Butterworth; Morgan (1968).

A azadiractina, principal ingrediente ativo presente na planta, atua repelindo os insetos, evitando A alimentação e postura de ovos nas plantas tratadas; atraso no crescimento devido à redução na alimentação e o crescimento dos insetos, dificultando o seu desenvolvimento; ovicida e larvicida, impedindo a eclosão dos imaturos, eliminando larvas de insetos; redução da fecundidade e fertilidade; indução de deformidades (SOGLIA et al; 2007).

Heyde et al. (1983) estudaram a utilização do óleo de nim como repelente em três espécies de pragas do arroz. Os resultados revelaram que menos de $8 \%$ dos insetos chegaram nas plantas tratadas, concluindo que o contato com o óleo de nim e seu odor repele insetos. Estes autores também concluíram que houve redução alimentar de tais pragas nas plantas tratadas com extratos e óleo de nim, até mesmo na menor 
concentração de óleo de nim (1\%). O extrato de nim foi usado por Néri et al. (2006), visando controlar a mosca-branca na cultura do melão e os autores observaram que em todas as parcelas onde o extrato aquoso de nim foi aplicado, os insetos foram repelidos.

Esse estudo teve por objetivo testar diferentes doses de nim, para o controle de Bemisia spp. em abobrinha italiana, na época da seca.

\section{MATERIAL E MÉTODOS}

O trabalho foi conduzido no Campus do Centro Universitário Moura Lacerda, localizado na cidade de Ribeirão Preto, SP, situada a 620 metros de altitude e a $21^{\circ} 10^{\prime} 04^{\prime \prime}$ de latitude sul e 4746'23" de longitude oeste. A classificação do clima é subtropical temperado, com média anual de $21^{\circ} \mathrm{C}$ de temperatura e precipitação média de $1.500 \mathrm{~mm}$ por ano.

A semeadura da cucurbitácea Cucurbita pepo L. var melopepo cv. Caserta (abobrinha italiana) ocorreu no dia 26 de junho de 2008, por semeadura direta, com espaçamento de 60 centímetros entre covas e duas sementes por cova. Não foi feita correção ou adubação no solo da área. A rega foi feita a cada três dias para manter a umidade do solo por meio de aspersores, e capinas foram realizadas periodicamente para controle de plantas daninhas. As plantas foram submetidas ao estresse hídrico por

7 dias, para que ficassem mais suscetíveis à praga favorecendo o aparecimento da mesma.

Os tratamentos foram distribuídos segundo o delineamento experimental de blocos casualizados, sendo constituído de seis tratamentos correspondendo às doses de produtos para o controle do inseto (testemunha sem aplicação, extrato de nim nas dosagens de $50,70,80,90 \mathrm{~mL}$ por $10 \mathrm{~L}$ de água e o produto comercial Actara (thiametoxan), na dosagem de $1,5 \mathrm{~g}$ por $10 \mathrm{~L}$ de água) e cinco repetições.

Foram usados dois pulverizadores costais da marca Strong, de 20L cada um. Um foi utilizado para aplicação do Actara e outro, certificado de que não haviam resíduos de outros produtos, para o nim.

Cada parcela foi constituída por 3 linhas de plantio, com $4 \mathrm{~m}$ de comprimento e 12 plantas por parcela.

Houve uma amostragem prévia, onde foi contado o número de insetos presentes em cada parcela experimental. A pulverização dos produtos só foi realizada após as amostragens prévias. 
O controle foi feito após a detecção da praga na área. Foram realizadas mais 4 avaliações da praga em 12 plantas por parcela 1 dia após, 3 dias após, 5 dias após e 7 dias após as aplicações dos tratamentos .

A redução média da quantidade de adultos e ninfas da mosca-branca foi calculada pela fórmula proposta por Henderson; Tilton (1955), como segue abaixo:

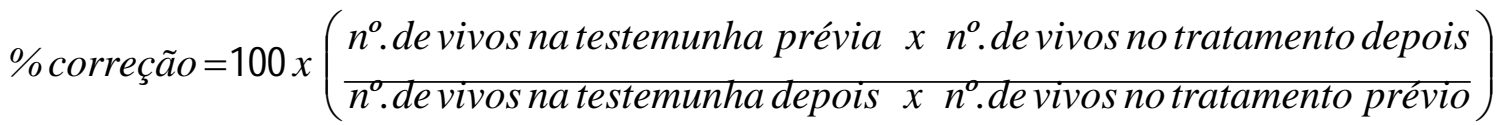

Todos os dados obtidos foram submetidos ao teste de homocedasticidade para a determinação da melhor forma de análise. As médias obtidas foram comparadas entre si pelo teste de Tukey, ao nível de 5\% de probabilidade.

\section{RESULTADOS E DISCUSSÃO}

A avaliação prévia do número de insetos por parcela (dia 0 após a aplicação) não apresentou diferenças significativas entre os tratamentos, indicando homogeneidade das parcelas (Tabela 2).

TABELA 2. Número médio de insetos de Bemisia tabaci por tratamentos por dias após as aplicações. Ribeirão Preto, SP, 2008.

\begin{tabular}{lccccc}
\hline \multirow{2}{*}{ Tratamentos } & \multicolumn{5}{c}{ Dias após a aplicação } \\
\cline { 2 - 6 } & $\mathbf{0}$ & $\mathbf{1}$ & $\mathbf{3}$ & $\mathbf{5}$ & $\mathbf{7}$ \\
\hline Testemunha & $4,2 \pm 0,6 \mathrm{a}^{1}$ & $5,0 \pm 0,7 \mathrm{~b}$ & $5,8 \pm 0,6 \mathrm{c}$ & $7,4 \pm 0,5 \mathrm{c}$ & $8,0 \pm 0,4 \mathrm{c}$ \\
Nim 50 & $4,0 \pm 0,4 \mathrm{a}$ & $1,0 \pm 0,3 \mathrm{a}$ & $1,8 \pm 0,4 \mathrm{~b}$ & $3,8 \pm 0,8 \mathrm{~b}$ & $4,2 \pm 0,7 \mathrm{~b}$ \\
Nim 70 & $3,0 \pm 0,7 \mathrm{a}$ & $0,6 \pm 0,2 \mathrm{a}$ & $1,4 \pm 0,2 \mathrm{ab}$ & $2,8 \pm 0,4 \mathrm{~b}$ & $3,2 \pm 0,4 \mathrm{~b}$ \\
Nim 80 & $3,2 \pm 0,6 \mathrm{a}$ & $0,4 \pm 0,2 \mathrm{a}$ & $1,2 \pm 0,3 \mathrm{ab}$ & $2,2 \pm 0,2 \mathrm{ab}$ & $3,0 \pm 0,3 \mathrm{~b}$ \\
Nim 90 & $3,6 \pm 0,4 \mathrm{a}$ & $0,4 \pm 0,0 \mathrm{a}$ & $1,0 \pm 0,2 \mathrm{ab}$ & $1,8 \pm 0,4 \mathrm{ab}$ & $2,6 \pm 0,2 \mathrm{ab}$ \\
Actara & $3,4 \pm 0,5 \mathrm{a}$ & $0,0 \pm 0,0 \mathrm{a}$ & $0,2 \pm 0,2 \mathrm{a}$ & $0,4 \pm 0,2 \mathrm{a}$ & $1,2 \pm 0,2 \mathrm{a}$ \\
\hline
\end{tabular}

T'Médias seguidas pela mesma letra, na coluna, não diferem entre si pelo teste de Tukey a $5 \%$ de significância.

No primeiro dia após a aplicação do extrato de nim e do thiamethoxan, pôdese observar que apenas a testemunha foi estatisticamente diferente dos demais tratamentos em relação ao número de moscas-brancas (Tabela 2). Todos os outros tratamentos sofreram redução no número de moscas-brancas. A redução de adultos 
foi superior a $80 \%$, sendo próxima de $100 \%$ na parcela tratada com thiameto xan (Figura 1). Foram observados, no

tratamento com o thiametoxan, adultos da praga, mortos ao redor das plantas, nas parcelas onde o produto foi aplicado.

Três dias após a aplicação, houve diferença significativa do tratamento (Figura 1) com nim à dosagem de $50 \mathrm{~mL} / 10 \mathrm{~L}$ de água, mostrando um aumento da população de Bemissia spp. nas parcelas com esse tratamento e testemunha respectivamente (Tabela 2)

No quinto dia após as aplicações foi observado um aumento significativo no número de moscas-brancas no tratamento com extrato de nim a $70 \mathrm{~mL} / 10 \mathrm{~L}$ de água, sendo que os tratamentos com $80 \mathrm{~mL} / 10 \mathrm{~L}$ de água e o de $90 \mathrm{~mL} / 10 \mathrm{~L}$ de água, sofreram um aumento no número de insetos, porém não significativo em relação ao tratamento com thiametoxan à dosagem de 1,5 g/10 L de água. (Tabela 2).

Ao sétimo dia de avaliação, notou-se que o tratamento com thiametoxan (Tabela 2), continuou mantendo a população de mosca-branca baixa, e estatisticamente diferente dos tratamentos com nim 80,70 e $50 \mathrm{~mL} / 10 \mathrm{~L}$ de água e a testemunha.

A redução média (\%) de mosca-branca nos tratamentos com nim e thiametoxan (Figura 1) seguiu a mesma tendência da testemunha no primeiro dia após sua aplicação. Todos os tratamentos resultaram em porcentagem média de redução superior a $80 \%$ nesse período. O tratamento com thiametoxan apresentou durante todo o período de avaliação, uma redução de acima de $80 \%$ dos insetos presentes. $\mathrm{O}$ tratamento com o extrato de nim nas dosagens de 90 e $80 \mathrm{~mL} / 10 \mathrm{~L}$ de água foram bastante semelhantes no primeiro dia após a aplicação, com uma redução de $90 \%$ na porcentagem de mosca-branca nas parcelas. Porém, à partir do terceiro dia de avaliação, somente a aplicação do extrato de nim na dosagem de $90 \mathrm{~mL} / 10 \mathrm{~L}$ água manteve a população de mosca-branca com uma redução de $80 \%$ do número de indivíduos. E no sétimo dia de avaliação, esta porcentagem já havia caído para pouco mais de $60 \%$ (Figura 1), mostrando que este produto não possui um poder residual comparável aos outros grupos de inseticidas considerados padrões (thiametoxan). $\mathrm{O}$ extrato de nim nas dosagens de 50 e $70 \mathrm{~mL} / 10 \mathrm{~L}$ de água a partir do quinto dia de avaliação, tiveram uma redução percentual da população de moscas brancas em relação a testemunha, de menos de $50 \%$, mostrando a inviabilidade de sua aplicação para o controle deste inseto na cultura da abobrinha. 
A diferença entre os tratamentos, para o número de adultos nas plantas, pode ser explicada pelo efeito repelente do extrato de nim e sobre a redução alimentar dos insetos, conforme relatado por Neri et al. (2006). Estes autores observaram que, na cultura do melão,

em todas as parcelas onde foi aplicado o extrato aquoso de nim, os insetos foram repelidos. Schmutterer (1990), em seu experimento com a praga do arroz Nilaparvata lugens (Hemiptera, Delphacidae), concluiu que poucos adultos desta praga pousaram sobre as plantas tratadas com óleo de nim no volume de $3 \%$, provando sua ação repelente. O estudo realizado por Heyde et al. (1983) mostrou que a ação de extratos e do óleo de nim em $N$. lugens, Sogatella furcifera (Hemiptera: Delphacidae) e Nephotettix virescens (Hemiptera: Cicadellidae), provocou significativa redução da alimentação de tais pragas, mesmo em concentração baixa, a $1 \%$.

Segundo Prabhaker et al. (1989), o extrato aquoso de nim possui maior efeito nos ovos do que na fase adulta de aleirodídeos, pois é na fase adulta que os insetos possuem maior resistência aos inseticidas, o que pode ser constatado no retorno de $B$. tabaci às plantas devido ao baixo poder residual do extrato de nim.

De acordo com o fabricante do extrato de nim, a dosagem que deve ser aplicada em canteiros e hortas comerciais é a de $700 \mathrm{~mL} / 100 \mathrm{~L}$ de água, para efeito curativo. Essa recomendação, não foi a que obteve melhores resultados no experimento, sendo a dosagem de $90 \mathrm{~mL} / 10 \mathrm{~L}$ de água $(900 \mathrm{~mL} / 100 \mathrm{~L}$ de água) obteve resultados expressivos em relação ao controle da mesma.

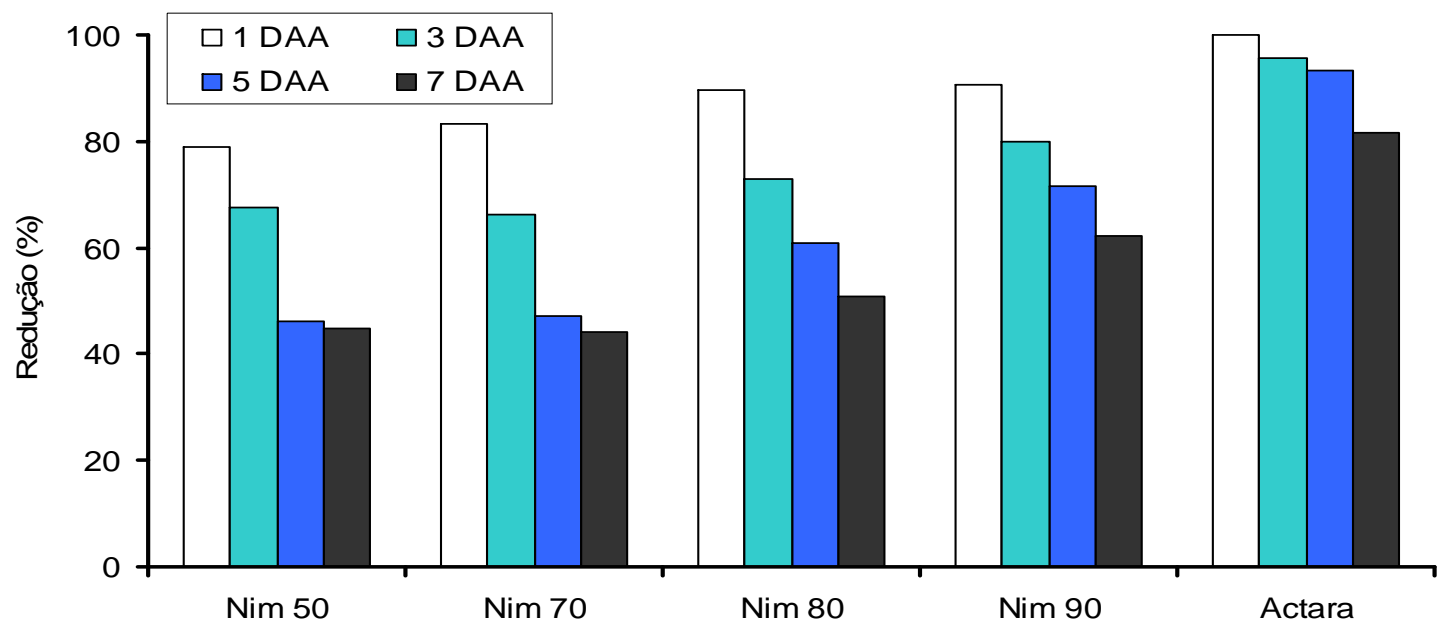

FIGURA 1. Redução média (\%) de moscas-brancas, Bemisia spp. após a aplicação de extrato de nim e thiametoxan. Ribeirão Preto, SP, 2008. 


\section{CONCLUSÃO}

Baseado nas condições em que o experimento foi conduzido, pode-se concluir que:

O extrato de nim usado nas dosagens de 50,70 e 80 e $90 \mathrm{~mL}$ de nim/10L de água obteve ação repelente e de redução alimentar do inseto, um dia após sua aplicação, mas devido ao baixo poder residual do extrato e às concentrações, ocorreu um aumento significativo no número de moscas-brancas com o decorrer dos dias.

A melhor dosagem para o controle de Bemisia tabaci na cultura da abobrinha (Cucurbita pepo var. melopepo) é de $900 \mathrm{~mL} / 100 \mathrm{~L}$ de água do extrato de nim.

\section{REFERÊNCIAS}

BEDFORD, I. D. et al. Geminivirus transmission and biological characterisation of Bemisia tabaci (Gennadius) byotypes from different geographic regions. Annal of Applied Biology, Warwick v.125, p. 311-325, 1994.

BROWN, J.K.; BIRD, J. Whitefly-transmitted geminiviruses and associated disorders in the Americas and Caribbean Basin. Plant Disease. Minnesota v..76, p.220-225, 1992.

BROWN, J.K. et al. The sweetpotato or silverleaf whiteflies : biotypes of Bemisia tabaci or a species complex ? Annual Review of Entomology, Palo Alto, v.40, p.511534, 1995.

BUTTERWORTH, J.H.; MORGAN, E.D. Isolation of a substance that suppresses feeding in locusts. Journal of the Chemical Society, Chemical Communication, London, v.35, n.1, p.23-24, 1968.

BYRNE, D. N.; BELLOWS, T. S. Whitefly biology. Annual Review of Entomology, Palo Alto, v.36, p.431-457, 1991.

CARValho, S. P.; MESQUita, A. C. Cultura da Abobrinha. Emater, 2001. Disponível em: http://www.emater.mg.gov.br/doc\%5Csite $\% 5$ Cserevicoseprodutos $\% 5$ Clivraria $\% 5 C O l e i$ cultura\%5CA\%20cultura\%20da\%20Abobrinha.pdf>. Acesso em 13 jan. 2008.

CIOCIOLA, J. A. L; MARTINEZ, S. S. Nim: alternativa no controle de pragas e doenças. Belo Horizonte: EPAMIG. Boletim Técnico n.67. 2002, 24 p.

COSTA, A.S. Whitefly-transmited plant diseases. Annual Review of Phytopathology, Palo Alto v.16, p.429-449. 1976. 
COSTA, H. S.; BROWN, J. K. Variation in biological characteristics and esterase patterns among populations of Bemisia tabaci, and the association o fone population with silverleaf symptom induction. Entomologia Experimentalis et Applicata, Dordrecht, v.61, p.211-219, 1991.

FRANÇA, F.H.; VILLAS BOAS, G.L.; BRANCO, M.C. Ocorrência de Bemisia argentifolii Bellows \& Perring (Homoptera: Aleyrodidae) no Distrito Federal. Anais da Sociedade Entomológica do Brasil, Londrina, v.25, n.2, p.369-372, 1996.

GALLO, D.; et al.. Entomologia agrícola. Piracicaba: FEALQ, 2002. 920 p. (Biblioteca de Ciências Agrárias Luiz de Queiroz, 10).

HAJI, F. et al. Circular Técnica, Manejo da Mosca-Branca na Cultura do Tomate. Embrapa Semi-árido, $1^{\circ}$ edição, 2005.

HENDERSON, C.F.; TILTON, E.W. Tests with acaricides against the brown wheat mite. Journal of Economic Entomology, Lanham, v.48, p.157-161, 1955.

HEYDE, J. V.D.; SAXENA, R.C., SCHUMUTTERER, H. Neem oil and neem extracts as potential insecticides for control of hemipterous rice pests. Proceedings of the Second International Neem Conference, Rauischholzhausen, 1983.

HILJE, L. Aspectos bioecológicos de Bemisia tabaci em Mesoamerica. Manejo Integrado de Plagas, Turrialba, n.35, p.46-54, 1995. Disponível em:

<http://www.biotropic.com.mx/upload/Conferencia-III-Seminario-Dr-Luko-Hilje.doc.> Acesso em: 13 jul. 2008.

LANA, M. M. et al. Abobrinha. Disponível em:

http://www2.correioweb.com.br/hotsites/alimentos/abobrinha/alimentos.htm. Acesso em 12 fev. 2008.

LENTEREN, J.C. V.; NOLDUS, P.J.J. Whitefly-plant relationships: behavioural and ecological aspects. In D. Gerling (ed), Whiteflies: their bionomics, pest status and management. Wimborne, Intercept, 348p. 1990.

LOURENÇÃO, A. L., NAGAI, H. Surtos populacionais de Bemisia tabaci no Estado de São Paulo. Brangantia, Campinas, v.53, p.53-59, 1994.

MATTOS, M. A. de A. Bemisia argentifolii Bellows \& Perring (Hemíptera: Aleyrodidae), na cultura do tomate no Submédio do Vale do São Francisco: estratégias de controle com agroquímicos, efeitos sobre a maturação irregular dos frutos, Brix, acidez, produtividade e análise do benefício/custo. 2001. $66 \mathrm{f}$. Dissertação (Mestrado) - Universidade Federal Rural de Pernambuco, Recife.

NERI, D. K. P.; et al. Efeito do extrato aquoso de nim sobre Bemisia tabaci Biotipo B (Gennadius) (Hemiptera: Aleyrodidae), em meloeiro. Revista Verde , Mossoró, v.1, n.2, p.48-53 julho/dezembro de 2006. 
NEVES, B. P.; OLIVEIRA, I. P.; NOGUEIRA, J. C. M. Cultivo e utilização do nim indiano. Santo Antonio de Goiás: Embrapa-CNPAF, 2003. 12p. (Embrapa - CNPAF. Circular técnica, 62).

OLIVEIRA, M.R.V. Alerta fitossanitário 1. Prevenção e controle da mosca-branca. 2001. Disponível em:

<http://www.agricultura.gov.br/portal/page?_pageid=33,7146662\&_dad=portal\&_sche ma=PORTAL>. Acesso em: 25 set 2008.

PRABHAKER, N.; TOSCANO, N.C.; COUDRIET, D.L. Susceptibility of the immature and adult stages of the sweetpotato whitefly (Homoptera: Aleyrodidae) to selected inseticides. Journal of Economic Entomology,1989. Disponível em:

<http://cat.inist.fr/?aModele=afficheN \&cpsidt=6630861 > . Acesso em: 12 mar. 2009.

SCHMUTTERER, H. Properties and potential of natural pesticides from the neem tree, Azadirachta indica. Annual Review of Entomology. Palo Alto, v.35, p.271-297, 1990.

SOGLIA, M. da C. et al. Usos e aplicação do nim (Azadirachta indica). Disponível em: <htpp//www.embrapa.gov.br>. Acesso em: 22 set 2007.

ZUCCHI, R.A., SILVEIRA NETO, S., NAKANO, O. Guia de identificação de pragas agrícolas. Piracicaba: FEALQ, 1993, 139p. 